\title{
Fluoretação da água no Brasil: distribuição regional e acurácia das informações sobre vigilância em municípios com mais de 50 mil habitantes
}

\section{Water fluoridation in Brazil: regional distribution and accuracy of information on surveillance in municipalities with more than 50,000 inhabitants}

\author{
Fluorización del agua en Brasil: distribución \\ regional y exactitud de la información sobre \\ su vigilancia en municipios con más de \\ 50 mil habitantes
}

Angelo Giuseppe Roncalli 1

Luiz Roberto Augusto Noro 1

Jaime Aparecido Cury ${ }^{2}$

Celso Zilbovicius 3

Helder Henrique Costa Pinheiro 4

Helenita Corrêa Ely 5

Paulo Capel Narvai 6

Paulo Frazão 6

\section{Resumo}

Dados de saneamento para 2008 indicavam que 74,4\% dos municípios com mais de 50 mil habitantes recebiam água fluoretada, entretanto, critérios para a validação desta informação não foram adotados. O estudo objetivou verificar a acurácia da informação sobre a fluoretação da água, tomando-se por referência dados de vigilância da água em municípios com mais de 50 mil habitantes no ano de 2008 e no período de 2010-2015. Dados de diferentes fontes foram empregados comparando-se as informações relativas ao período. Amostras da água foram coletadas e a concentração de fluoreto foi obtida pelo método eletrométrico. O processo de verificação ocorreu pela confrontação dos dados obtidos por diferentes fontes. Seiscentos e um municipios (97,9\%) tiveram os dados confrontados. A taxa de municípios que realizam vigilância com base no heterocontrole passou de 39,4\% para 48,5\%. Observou-se elevada taxa de falsos positivos ou negativos $(15,1 \%)$ em relação aos dados de 2008. A cobertura municipal foi de 70,2\%, 4,2 pontos abaixo da estimativa $(74,4 \%)$ divulgada para 2008. Registros da prática da vigilância foram observados em 54,3\% dos municípios fluoretados, cuja cobertura populacional alcança 50\% ou mais da população que recebe água tratada. Houve sensível melhoria nos dados de vigilância em relação ao fluoreto, embora com porcentual importante de falsos positivos e negativos. Ainda são largas as diferenças inter-regionais relacionadas tanto à provisão da fluoretação da água quanto à implementação da vigilância nos municípios pesquisados, trazendo importantes desafios para a saúde pública.

Fluoretação; Saúde Bucal; Vigilância Sanitária

\author{
Correspondência \\ A. G. Roncalli \\ Departamento de Odontologia, Centro de Ciências da Saúde, \\ Universidade Federal do Rio Grande do Norte. \\ Av. Salgado Filho 1787, Natal, RN 59056-000, Brasil. \\ roncalli@terra.com.br \\ 1 Centro de Ciências da Saúde, Universidade Federal do Rio \\ Grande do Norte, Natal, Brasil. \\ 2 Faculdade de Odontologia de Piracicaba, Universidade \\ Estadual de Campinas, Piracicaba, Brasil. \\ 3 Faculdade de Odontologia, Universidade de São Paulo, São \\ Paulo, Brasil. \\ 4 Faculdade de Odontologia, Universidade Federal do Pará, \\ Belém, Brasil. \\ 5 Pontifícia Universidade Católica do Rio Grande do Sul, Porto \\ Alegre, Brasil. \\ 6 Faculdade de Saúde Pública, Universidade de São Paulo, São \\ Paulo, Brasil.
}




\section{Introdução}

Dados sobre água, saneamento e qualidade do ar mostram que é necessário fortalecer os sistemas de vigilância e que o aproveitamento das tecnologias de informação e comunicação pode melhorar estes sistemas e ampliar a sua utilização em vários níveis da sociedade 1 . Com isso, as tecnologias digitais de saúde poderão desempenhar um papel importante na realização das Metas de Desenvolvimento Sustentável 2.

Entre as iniciativas para aprimorar os sistemas de vigilância em saúde destaca-se o esforço relacionado aos registros de mortalidade. A taxa de óbitos por causas mal definidas era relativamente alta no Brasil, com grandes diferenças regionais. Em meados dos anos 2000, um projeto de qualificação da informação reduziu consideravelmente essas taxas, melhorando a qualidade da informação ${ }^{3}$. A despeito de algumas diferenças regionais, esse esforço tem proporcionado uma melhoria crescente dos registros 4 , demonstrando que a qualidade da informação deve também ser objeto de políticas públicas.

A necessidade do compartilhamento de dados e ampliação da produção e disseminação de informações de saúde de forma a atender tanto às necessidades de usuários, profissionais, gestores, prestadores de serviços e controle social, quanto ao intercâmbio com instituições de ensino e pesquisa, outros setores governamentais e da sociedade e instituições internacionais foi enfatizada pela 1a Conferência Nacional de Vigilância em Saúde, cuja etapa nacional foi realizada em Brasília, de 27 de fevereiro a 02 de março de 20185 .

A relevância da água como determinante ambiental 6 redundou na necessidade de revisão de paradigmas no sentido de possibilitar uma abordagem que englobasse todas as etapas do fornecimento da água, incluindo desde a captação até o uso pelo consumidor final 7 . Há um crescente reconhecimento de que o fortalecimento dos sistemas de vigilância da qualidade da água exige a colaboração de uma rede de atores e alianças envolvendo diferentes departamentos, setores e organizações que permita a integração de dados e a produção de informações úteis para a tomada de decisão 8 .

Entre os parâmetros para a determinação dos padrões de qualidade da água destaca-se o fluoreto (íon flúor). De modo análogo a outros parâmetros como turbidez, cloro residual, colimetria, $\mathrm{pH}$, agrotóxicos e mercúrio, o fluoreto é um importante indicador porque os valores da sua concentração podem representar proteção ou risco à saúde da dentição. $\mathrm{O}$ ajuste da sua concentração para fins de prevenção da cárie dentária em nível populacional é uma importante tecnologia de saúde pública recomendada por eminentes instituições científicas, sanitárias e profissionais ao redor do mundo em decorrência de sua efetividade e segurança 9 . Embora a investigação do grau de acurácia das informações sobre a concentração de fluoreto nas águas de abastecimento público seja relevante para a identificação da provisão da fluoretação da água e dos avanços e gargalos dos sistemas de informação e de vigilância em relação ao parâmetro fluoreto, um importante indicador da qualidade da água 10, nenhuma pesquisa verificou a acurácia destas informações em dois momentos no tempo.

O objetivo deste estudo foi verificar a acurácia da informação sobre a fluoretação da água de abastecimento público, tomando-se por referência dados de vigilância da água em municípios brasileiros com mais de 50 mil habitantes no ano de 2008 e no período de 2010-2015.

\section{Método}

Os dados utilizados no presente trabalho foram obtidos com base no projeto de pesquisa Cobertura $e$ Vigilância da Fluoretação da Água de Abastecimento Público no Brasil, 2010-2015, também conhecido como "Projeto Vigifluor" (detalhes em http://www.cecol.fsp.usp.br/). Informações sobre a situação quanto à fluoretação e sobre a concentração de fluoreto na água de abastecimento público provenientes de diferentes fontes documentais foram empregadas, comparando-as em 2008 e entre 2010 e 2015. Em municípios onde não se dispunham de informações, amostras do sistema de abastecimento de água foram coletadas durante três meses para determinar a concentração de fluoreto.

A pesquisa teve abrangência nacional e as unidades do estudo foram compostas por todos os municípios brasileiros com mais de 50 mil habitantes no ano de 2012. Esses municípios abrangem cerca de $65 \%$ da população brasileira e constituem polos de regiões de saúde que projetam sua influência 
sanitária estratégica para os municípios de menor porte demográfico das respectivas regiões. Fazem parte da primeira etapa de uma pesquisa mais abrangente que, em etapa posterior, poderá possibilitar o aprofundamento e detalhamentos de informações para a totalidade dos municípios brasileiros que está distribuída nas 27 Unidades da Federação, em cinco regiões.

As fontes documentais foram a Pesquisa Nacional de Saneamento Básico (PNSB) promovida em 2008 pela Fundação Instituto Brasileiro de Geografia e Estatística 11 e os relatórios técnicos sobre cobertura e vigilância da fluoretação de águas de abastecimento público, disponíveis pelo Sistema de Informação de Vigilância da Qualidade da Água para Consumo Humano (SISAGUA), mantido pelo Programa Nacional de Vigilância em Saúde Ambiental relacionada à qualidade da água para o consumo humano 12 .

Nos municípios onde a informação foi considerada não validada por mais de um tipo de fonte, a concentração de fluoreto na água foi investigada mediante análise laboratorial. Os pontos de coleta no município selecionado foram estabelecidos conforme o Guia de Amostragem para Vigilância da Concentração do Fluoreto na Água de Abastecimento Público, disponibilizado pelo Centro Colaborador do Ministério da Saúde em Vigilância da Saúde Bucal 13.

Os procedimentos técnicos adotados seguiram o Protocolo de Coleta e Análise da Amostra de Água, disponibilizado pelo Centro Colaborador do Ministério da Saúde em Vigilância da Saúde Bucal 13. A análise foi realizada pelo método eletrométrico, pela técnica direta, devido à sua praticidade, precisão e exatidão, no Laboratório de Bioquímica da Faculdade de Odontologia de Piracicaba, Universidade Estadual de Campinas, São Paulo, Brasil (FOP-UNICAMP).

Um banco de dados foi construído com as seguintes variáveis: região; estado; município; situação quanto à fluoretação segundo a PNSB-2008 (sim ou não); valor médio da concentração de fluoreto e população coberta por água fluoretada segundo o SISAGUA. O valor médio da concentração de fluoreto foi obtido para o ano de 2008 e para os diferentes anos no período de 2010 a 2015. Foram considerados apenas os valores médios provenientes de municípios com quatro ou mais meses de registro de dados 14 .

Os valores médios foram classificados em sete categorias (1 - não cadastrado; 2 - não informado; 3 - 0,000 a 0,344mg F/L; 4 - 0,345 a 0,444mg F/L; 5 - 0,445 a 0,944mg F/L; 6 - 0,945 a 1,544mg F/L; 7 - > 1,544mg F/L) conforme documento de consenso técnico 15.

Os dados primários provenientes da análise laboratorial foram classificados da mesma forma. A população coberta por água fluoretada foi obtida para ano de 2014.

Os dados foram analisados classificando-se os meios de confrontação empregados em seis categorias mutuamente exclusivas: 1 - apenas documentação obtida em 2008; 2 - apenas documentos de 2010-2015; 3 - documentos do SISAGUA 2008 e do SISAGUA 2010-2015; 4 - apenas dados laboratoriais de 2014-2015; 5 - dados laboratoriais de 2014-2015 e documentos do SISAGUA 2008 e 2010-2015; e 6 - somente dados de cobertura populacional. Tomando-se como referência os dados do SISAGUA relativos ao ano de 2008 e ao período de 2010-2015, a informação sobre a provisão da política pública foi comparada com os dados oriundos da PNSB-2008, para cada município, identificando-se as situações nas quais a informação fornecida pela PNSB registrada para 2008 representava um valor verdadeiro positivo ou negativo, ou um valor falso positivo ou negativo em relação aos dados do SISAGUA. As frequências absoluta e relativa desses valores de acurácia foram apuradas para cada região. Embora dados da PNSB-2008 tenham sido confrontados com os dados relativos ao período de 2010 a 2015, este processo de verificação foi considerado lícito, admitindo-se que uma das premissas desta política pública para produzir os efeitos esperados é sua longitudinalidade, isto é, não haver solução de continuidade. A condição mais adequada é, portanto, aquela em que as ações de vigilância dispõem de dados oriundos do SISAGUA, que tem como objetivo auxiliar o gerenciamento de riscos à saúde associados à qualidade da água destinada ao consumo humano no âmbito do Sistema Único de Saúde (SUS) 16.

A vigilância da fluoretação pode ser feita apenas com base em dados do controle operacional, como ocorre em vários países, como os Estados Unidos. A experiência brasileira, de mais de meio século, indica, contudo, a insuficiência dessa estratégia de vigilância por razões que não podem ser aprofundadas neste estudo. Dessa experiência resultou a emergência entre nós do conceito de heterocontrole, seja para suprir deficiências do controle operacional, seja porque, contemporaneamente, o parâmetro fluoreto deve ser objeto de vigilância de qualquer água utilizada para o consumo humano, 
haja ou não sistema de fluoretação. Trata-se de medida cautelar em contextos em que não se pode assegurar que a população não esteja sendo exposta à água hiperfluoretada. Por esse motivo, neste estudo, o objetivo recai, exclusivamente, para as situações em que há, comprovadamente, vigilância com base em algum tipo de heterocontrole, independentemente de suas características operacionais, como plano de amostragem e opções analíticas, dentre outras.

Em relação à fluoretação, o município foi classificado em cinco categorias: 1 - não fluoretado; 2 - parcialmente fluoretado e sem vigilância (quando a cobertura era menor do que 50\% da população que recebe água tratada); 3 - parcialmente fluoretado com vigilância; 4 - fluoretado e sem vigilância (quando a cobertura era maior do que 49,9\% da população que recebe água tratada); 5 - fluoretado com vigilância. A presença de vigilância foi definida baseando-se em dados registrados de heterocontrole, ou seja, aqueles obtidos por instituições ou agências não responsáveis pelo tratamento/ fluoretação da água de abastecimento público 17, uma pré-condição importante para a qualificação dos sistemas de fluoretação 18 . Foram considerados municípios fluoretados com prática de vigilância aqueles que apresentavam quatro ou mais meses de registros de concentração de fluoreto na água de abastecimento, em dois ou mais anos, durante o período de 2010-2015, com valores médios incluídos nos intervalos de concentração correspondentes às categorias 4 a $6(0,345$ a $0,444 \mathrm{mg} F / L ; 0,445$ a 0,944mg F/L; 0,945 a 1,544mg F/L).

\section{Resultados}

Em 2012, havia 614 municípios com mais de 50 mil habitantes no país. Do total, 601 (97,9\%) tiveram os dados confrontados. Desses, foram empregadas fontes documentais de heterocontrole para 373 $(62,1 \%)$ municípios, distribuídos em fontes relativas ao ano de 2008, ao período de 2010-2015 e a ambos (Tabela 1). As categorias 1 e 3 da Tabela 1 agrupam municípios com dados de heterocontrole relativos ao ano de 2008, e as categorias 2 e 3 incluem municípios com dados relativos ao período de 2010 a 2015. Conforme a Tabela 1, o número de municípios que realiza a vigilância com base em dados de heterocontrole, uma pré-condição importante para a qualificação dos sistemas de fluoretação, passou de $242(39,4 \%)$ para $298(48,5 \%)$.

Comparando os períodos, houve sensível melhoria nas fontes de dados secundários para todas as regiões, excetuando-se a Centro-oeste. A porcentagem de municípios com dados no SISAGUA aumentou de $45,8 \%$ para $78,3 \%$. Considerando a provisão da fluoretação da água, a taxa de falsos positivos ou negativos manteve-se elevada em relação aos dados oficiais de saneamento para 2008, passando de $12,1 \%$ no primeiro período para $15,1 \%$ no segundo (Tabela 2 ).

A provisão municipal da política pública foi de 70,2\%, 4,2 pontos percentuais abaixo da estimativa (74,4\%) divulgada para 2008 pela PNSB. Considerando o nível de cobertura de $50 \%$ ou mais da popu-

Tabela 1

Número de municípios com validação concluída segundo as fontes de confrontação dos dados por região. Brasil, $2008-2015$.

\begin{tabular}{|c|c|c|c|c|c|c|c|c|c|c|}
\hline \multirow[t]{2}{*}{ Fonte de dados } & \multicolumn{2}{|c|}{ Norte } & \multicolumn{2}{|c|}{ Nordeste } & \multicolumn{2}{|c|}{ Sudeste } & \multicolumn{2}{|c|}{ Sul } & \multicolumn{2}{|c|}{ Centro-oeste } \\
\hline & $\mathbf{n}$ & $\%$ & $\mathbf{n}$ & $\%$ & $\mathbf{n}$ & $\%$ & $\mathbf{n}$ & $\%$ & $\mathbf{n}$ & $\%$ \\
\hline 1) Apenas documentos obtidos em 2008 (SISAGUA 2008) & 2 & 3,8 & 27 & 15,8 & 42 & 17,4 & - & 0,0 & 4 & 11,4 \\
\hline 2) Apenas documentos de 2010-2015 (SISAGUA 2010-2015) & 1 & 1,9 & 20 & 11,7 & 97 & 40,3 & 11 & 10,9 & 2 & 5,7 \\
\hline 3) Documentos do SISAGUA 2008 e 2010-2015 & - & 0,0 & 34 & 19,9 & 39 & 16,2 & 89 & 88,1 & 5 & 14,3 \\
\hline 4) Apenas dados laboratoriais de 2014-2016 & 36 & 67,9 & 27 & 15,8 & 14 & 5,8 & - & 0,0 & 5 & 14,3 \\
\hline 5) Dados laboratoriais de 2014-2016 e documentos do & 4 & 7,5 & 6 & 3,5 & 13 & 5,4 & - & 0,0 & 15 & 42,9 \\
\hline \multicolumn{11}{|l|}{ SISAGUA 2008 e 2010-2015 } \\
\hline 6) Somente dados de cobertura & 10 & 18,9 & 57 & 33,3 & 36 & 14,9 & 1 & 1,0 & 4 & 11,4 \\
\hline Total & 53 & 100,0 & 171 & 100,0 & 241 & 100,0 & 101 & 100,0 & 35 & 100,0 \\
\hline
\end{tabular}

SISAGUA: Sistema de Informação de Vigilância da Qualidade da Água para Consumo Humano. 
Tabela 2

Número de municípios segundo as categorias de acurácia para fluoretação da água e o período de tempo por região. Brasil, 2008-2015.

\begin{tabular}{|c|c|c|c|c|c|c|c|c|c|c|c|c|}
\hline & \multicolumn{2}{|c|}{ Norte } & \multicolumn{2}{|c|}{ Nordeste } & \multicolumn{2}{|c|}{ Sudeste } & \multicolumn{2}{|c|}{ Sul } & \multicolumn{2}{|c|}{ Centro-oeste } & \multicolumn{2}{|c|}{ Total } \\
\hline & $\mathbf{n}$ & $\%$ & n & $\%$ & $\mathbf{n}$ & $\%$ & n & $\%$ & $\mathbf{n}$ & $\%$ & n & $\%$ \\
\hline Total & 63 & 100,0 & 174 & 100,0 & 241 & 100,0 & 101 & 100,0 & 35 & 100,0 & 614 & 100,0 \\
\hline \multicolumn{13}{|l|}{2008} \\
\hline Sem informação & 58 & 92,1 & 108 & 62,1 & 146 & 60,6 & 11 & 10,9 & 10 & 28,6 & 333 & 54,2 \\
\hline Com informação * & 5 & 7,9 & 66 & 37,9 & 95 & 39,4 & 90 & 89,1 & 25 & 71,4 & 281 & 45,8 \\
\hline Falso negativo & - & 0,0 & 1 & 1,5 & 1 & 1,0 & 1 & 1,1 & - & 0,0 & 3 & 1,1 \\
\hline Falso positivo & - & 0,0 & 9 & 13,6 & 17 & 17,9 & 2 & 2,2 & 3 & 12,0 & 31 & 11,0 \\
\hline Verdadeiro negativo & 2 & 40,0 & 8 & 12,1 & - & 0,0 & - & 0,0 & - & 0,0 & 10 & 3,6 \\
\hline Verdadeiro positivo & 3 & 60,0 & 48 & 72,8 & 77 & 81,1 & 87 & 96,7 & 22 & 88,0 & 237 & 84,3 \\
\hline \multicolumn{13}{|l|}{ 2010-2015 } \\
\hline Sem informação & 10 & 15,9 & 3 & 1,7 & - & 0,0 & - & 0,0 & - & 0,0 & 13 & 2,1 \\
\hline Com informação ** & 53 & 84,1 & 171 & 98,3 & 241 & 100,0 & 101 & 100,0 & 35 & 100,0 & 601 & 97,9 \\
\hline Fonte primária & 40 & 75,5 & 33 & 19,3 & 27 & 11,2 & - & 0,0 & 20 & 57,1 & 120 & 20,0 \\
\hline Fonte secundária & 13 & 24,5 & 138 & 80,7 & 214 & 88,8 & 101 & 100,0 & 15 & 42,9 & 481 & 80,0 \\
\hline Falso negativo & 1 & 1,9 & 10 & 5,8 & 4 & 1,7 & 1 & 1,0 & - & 0,0 & 16 & 2,6 \\
\hline Falso positivo & 15 & 28,3 & 21 & 12,2 & 21 & 8,7 & 10 & 9,9 & 8 & 22,9 & 75 & 12,5 \\
\hline Verdadeiro negativo & 33 & 62,3 & 77 & 45,0 & 18 & 7,5 & - & 0,0 & 5 & 14,3 & 131 & 21,8 \\
\hline Verdadeiro positivo & 4 & 7,5 & 65 & 38,0 & 198 & 82,1 & 90 & 89,1 & 22 & 62,9 & 379 & 63,1 \\
\hline
\end{tabular}

SISAGUA: Sistema de Informação de Vigilância da Qualidade da Água para Consumo Humano.

* Diz respeito a municípios com informação extraída com base em dados do SISAGUA;

** As categorias representam situações decorrentes da verificação da informação fornecida pela Pesquisa Nacional de Saneamento Básico registrada para 2008, tomando-se por referência os dados do SISAGUA.

lação que recebe água tratada, a taxa decresceu para $62,7 \%, 11,7$ pontos percentuais aquém da estimativa para 2008, com importantes diferenças regionais (Figura 1). Quando se considera a cobertura em termos populacionais, esses valores aumentam, sendo coberta pela medida $78,6 \%$ da população (Figura 2). Os municípios com práticas de fluoretação são também os mais populosos e as diferenças regionais continuam marcantes.

Levando-se em conta os municípios fluoretados cuja cobertura populacional alcança 50\% ou mais da população que recebe água tratada, registros da prática da vigilância foram observados em 54,3\% deles, sendo as taxas mais elevadas nas regiões Sudeste e Sul, em comparação com as demais (Figura 2).

\section{Discussão}

Com base em diferentes fontes de informação dos dados de concentração de fluoreto na água de abastecimento público, este trabalho analisou a acurácia das informações sobre a provisão da fluoretação da água e a vigilância valendo-se de dados de heterocontrole ${ }^{17}$. Os resultados mostraram importantes diferenças entre os municípios segundo as regiões brasileiras.

A situação de melhor desempenho em termos de informação foi observada na Região Sul, onde cerca de 9 em cada 10 municípios apresentavam dados registrados no Sisagua tanto para o ano de 2008 quanto para o período 2010-2015. Na Região Nordeste, aproximadamente 1/3 dos municípios apresentava apenas dados relativos à cobertura, sem qualquer característica de vigilância; ao passo que na Região Norte, em sete de cada dez municípios as informações foram obtidas somente com base em coleta de amostras de água realizada na presente pesquisa.

Outra importante constatação deste estudo diz respeito à magnitude da taxa de falsos positivos/negativos. Uma pesquisa em municípios brasileiros apontava a possibilidade de algum grau de 


\section{Figura 1}

Distribuição percentual do número de municípios com mais de 50 mil habitantes segundo a situação quanto à fluoretação por região. Brasil, $2010-2015$.

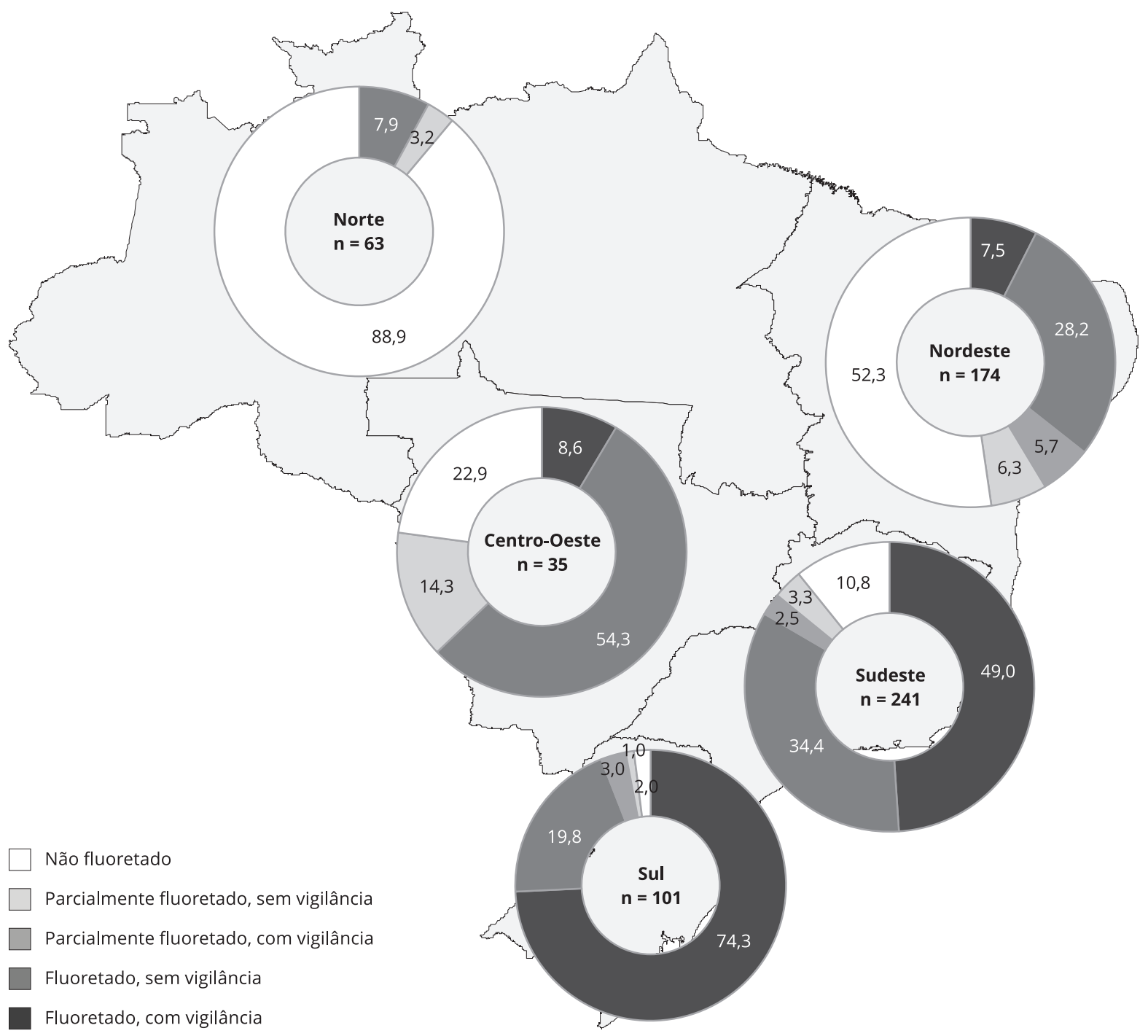

superestimação das taxas, dado que a situação da fluoretação tinha sido obtida apenas por meio de um questionário junto às entidades prestadoras dos serviços de saneamento ${ }^{13}$. Os resultados confirmaram essa hipótese, indicando mais de $10 \%$ de falsos positivos. Considerando que se trata de uma política intersetorial de saúde, aprovada em Lei pelo Congresso Nacional, uma variação dessa magnitude sugere fragilidade, tanto da administração pública municipal, órgão titular responsável pela concessão dos serviços de tratamento de água, quanto das formas de gestão setorial, participação social e de controle da política pública.

Os achados mostraram importante falta de provisão da fluoretação em municípios com mais de 50 mil habitantes nas regiões Norte $(88,9 \%)$ e Nordeste (52,3\%), evidenciando a omissão das autoridades em relação à previsão legal de fluoretação desde 1974. Considerando serem esses os municípios em melhores condições gerais, pode-se estimar as consequências para essas regiões como um todo. É 


\section{Figura 2}

Distribuição percentual da população em municípios com mais de 50 mil habitantes segundo a situação quanto à fluoretação por região. Brasil, $2010-2015$.

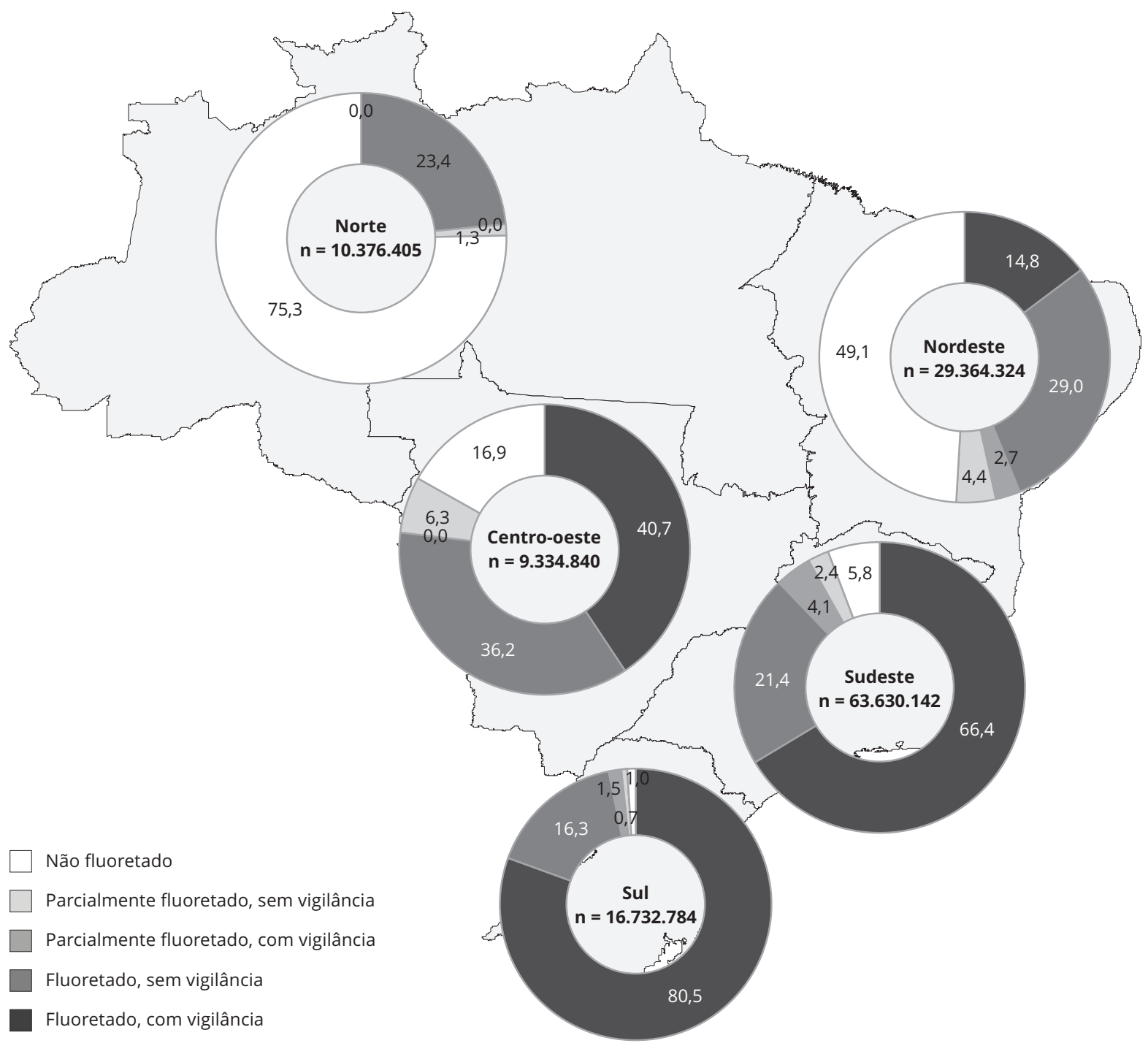

nítida a diferença entre a taxa de cobertura populacional de municípios fluoretados com vigilância nas regiões Sul (80,5\%) e Sudeste (66,4\%), em comparação com as regiões Nordeste $(14,8 \%)$ e Norte (0\%), ou seja, além da falta de fluoretação, a vigilância também aparece pobremente implantada nas regiões menos desenvolvidas.

Mudanças no nível de desigualdade da provisão da política pública foram observadas no início do século XXI, e guardam forte relação com as características que marcaram a trajetória do saneamento no país 13 . No Brasil, as políticas públicas representadas por serviços essenciais como energia elétrica, abastecimento de água, esgoto e coleta de lixo, alcançaram coberturas relevantes, primeiro nas regiões Sudeste e Sul, em comparação com as demais, conferindo uma trajetória de desigualdades territoriais 19 . Os resultados deste estudo confirmaram a influência que essa trajetória projeta tanto para as fontes de informação, quanto para a provisão da fluoretação da água e o sistema de vigilância, indicando a 
necessidade da formulação de estratégias que impulsionem a implementação das políticas públicas nas regiões Centro-oeste, Nordeste e Norte, considerando que a previsão da fluoretação da água de abastecimento público existe há mais de 40 anos 20 .

Em relação à vigilância, a comparação entre os dois períodos mostrou um importante aumento no número de municípios que a realizavam com base em dados de heterocontrole 17. Estudos anteriores de abrangência nacional produziram informações apenas para um único momento no tempo 14, e este é o primeiro trabalho que avaliou os registros de vigilância numa perspectiva longitudinal.

Nessa mesma perspectiva, dados de controle operacional do Sistema de Relatórios da Fluoretação da Água (Water Fluoridation Reporting System) mantidos pelo Centers for Disease Control and Prevention foram analisados entre o período de 2006-2010 e 2015, abrangendo 34 unidades federadas dos Estados Unidos da América 21.

A relevância do controle do nível de fluoreto para a qualidade da água e a importância da ação da vigilância ser realizada por meio da observação direta de amostras de água oriundas da rede de distribuição são reconhecidas por uma longa história de investigações 18, e estão presentes tanto nas diretrizes globais de qualidade da água 7, quanto nos dispositivos normativos aprovados pelo Ministério da Saúde em nosso país, cabendo destacar que a responsabilidade das autoridades sanitárias municipais pela vigilância, incluindo a implementação de plano próprio de amostragem, foi estabelecida em 2000 22, e desde então vem sendo mantida nos instrumentos de regramento da ação da vigilância. Mais recentemente, em julho de 2018, foi aprovada a Resolução no 588/2018 23 do Conselho Nacional de Saúde (CNS) que institui a Política Nacional de Vigilância em Saúde (PNVS), como fruto dos debates ocorridos na 1a Conferência Nacional de Vigilância em Saúde, que recomendou o aprimoramento dos sistemas de informação em saúde, bem como o acesso oportuno às análises dos dados por eles armazenados 5. A Resolução no 588/2018 traz atribuições específicas para municípios, estados e a União no campo da Vigilância em Saúde, a qual articula as ações das vigilâncias ambiental, sanitária e epidemiológica, além da vigilância em saúde do trabalhador. Dentre as atribuições municipais, o inciso VIII do Artigo 13 destaca que o município deve "regular, controlar, avaliar e fiscalizar procedimentos, produtos, substâncias e serviços de saúde e de interesse para a saúde” 23. Além disso, o relatório final da referida Conferência destaca a importância de rever os parâmetros de potabilidade da água, incluindo a necessidade de pesquisar o teor de flúor 24.

A experiência brasileira tem mostrado que o sistema de vigilância com base em dados de heterocontrole traz efeitos positivos para a qualidade da fluoretação da água de abastecimento público 18. De modo análogo ao Brasil, os Estados Unidos são um país de grande extensão territorial, longa tradição na implementação e elevada cobertura populacional da medida, entretanto, difere pelo fato de que, embora exista a possibilidade da observação direta de amostras provenientes da rede de distribuição nas normas de regulação da qualidade da água 25 , a vigilância em relação ao parâmetro fluoreto é realizada usualmente por meio de inspeção e auditoria junto às empresas responsáveis pelo tratamento da água, que realizam o controle tanto na saída do tratamento, quanto na rede de distribuição 26. Por estar embasada em dados de controle operacional, essa forma de vigilância empregada por muitas instituições é vulnerável a registros que não refletem a realidade, conforme descrito na literatura 18 .

Nos Estados Unidos, um estudo das condições de fluoretação da água do Estado de New Hampshire no período de 2000-2002 concluiu que níveis subótimos era o problema mais comum e que os sistemas de abastecimento precisavam melhorar o ajuste dos níveis de fluoreto para as comunidades aproveitarem todos os benefícios da fluoretação 27. Uma pesquisa em 2015 nos Estados Unidos em mais de 4 mil sistemas de abastecimento de água mostrou que 45\% dos sistemas ajustados mantiveram médias em relação à meta de $0,7 \mathrm{mg} \mathrm{F} / \mathrm{L}$, dentro de uma variação aceitável $( \pm 0,1 \mathrm{mg} \mathrm{F} / \mathrm{L})$ para ao menos 9 meses no ano, concluindo-se que faixas de controle mais estreitas que $\pm 0,2 \mathrm{mg} / \mathrm{L}$ poderiam ser viáveis para a concentração média mensal de fluoreto 21 .

Os achados do presente estudo, ao mesmo tempo em que indicaram relativa melhoria no sistema de informação, com exceção da Região Centro-oeste, mostraram que há um amplo espaço para o planejamento e desenvolvimento de atividades visando à expansão da ação da vigilância em cerca de metade dos municípios com mais de 50 mil habitantes. Uma pesquisa mostrou que a falta de cadastro e de alimentação do Sistema de Informação de Vigilância da Qualidade da Água de Consumo Humano estava associada a municípios com os piores indicadores sanitários, econômicos e de desenvolvimento humano ${ }^{14}$. Nos Estados Unidos, falta de recursos, especificamente de pessoal e tempo, foi identificada 
como barreira à participação no Water Fluoridation Reporting System 28. No que pesem esses aspectos, o progresso na informação sobre o parâmetro fluoreto no sistema de vigilância da água no Brasil pode representar uma importante inovação social no campo da política pública da fluoretação da água.

Reconhece-se que um dos principais desafios para o avanço da vigilância em saúde pública no século XXI diz respeito ao acesso e uso de dados. Analistas enxergam oportunidades para que provedores, instituições e agências de saúde pública compartilhem dados de interesse da saúde sem violar a confidencialidade e as prerrogativas do administrador, que continua sendo o responsável por garantir este e outros fatores como a qualidade e a visibilidade da informação 29.

Uma característica importante deste estudo diz respeito ao uso de diferentes fontes de dados. As limitações relacionadas aos registros foram mostradas e poderão servir de estímulo com vistas ao aprimoramento do sistema de informação. Embora a efetividade da fluoretação se mantenha 9 , a tecnologia de saúde pública tem sido questionada por iniciativas sem embasamento científico 13 , mas que têm respaldo em outros interesses, levando a iniciativas parlamentares, as quais segundo Zilbovicius et al. 30, buscam a revogação da lei que obriga a fluoretação em todos os municípios com estação de tratamento de água. Tal medida, sem dúvida, denotaria atraso na proteção que o Estado brasileiro deveria garantir em relação a um dos maiores benefícios coletivos, proporcionado por uma medida simples e eficaz como a fluoretação da água.

\section{Conclusão}

Embora tenha havido sensível melhoria nos dados do sistema de vigilância da água em relação ao parâmetro fluoreto para todas as regiões, excetuando-se a Centro-oeste, a persistência da desigualdade proporcionada pela perspectiva territorial fica evidenciada no presente estudo ao sinalizar a baixa provisão da fluoretação e sua vigilância quando se compara as regiões Norte e Nordeste com as regiões Sul e Sudeste. Considerando-se que foram incluídos no trabalho os municípios com mais de 50 mil habitantes e que a obrigatoriedade de fluoretação da água está prevista há mais de quarenta anos, essa discrepância fica ainda mais grave.

Confirmando as largas diferenças inter-regionais relacionadas à implantação efetiva dos sistemas de vigilância da fluoretação da água, observa-se na Região Sul a efetividade desta medida desde 2008, enquanto na Região Nordeste quase $40 \%$ dos municípios apresentaram apenas dados de cobertura, ao passo que na Região Norte em $70 \%$ dos municípios a coleta proveniente desta pesquisa foi a primeira iniciativa de monitoramento da fluoretação.

A fragilidade observada nas ações de vigilância da fluoretação em grande parte dos municípios pesquisados e os interesses escusos pautados na interrupção da fluoretação das águas trazem importantes desafios para as autoridades de saúde pública no século XXI, na proteção que o Estado brasileiro deveria assegurar em relação a um dos maiores benefícios coletivos, proporcionado por uma medida simples e eficaz, como a fluoretação da água. 


\section{Colaboradores}

A. G. Roncalli e P. Frazão contribuíram na concepção geral do projeto de pesquisa, concepção geral do artigo, escrita e revisão do texto. L. R. A. Noro, J. A. Cury e P. C. Narvai contribuíram na concepção geral do projeto de pesquisa, escrita e revisão do texto. C. Zilbovicius, H. H. C. Pinheiro e H. C. Ely contribuíram na concepção geral do projeto de pesquisa, coleta de dados e aprovação da versão final do manuscrito.

\section{Informações adicionais}

ORCID: Angelo Giuseppe Roncalli (0000-00015311-697X); Luiz Roberto Augusto Noro (00000001-8244-0154); Jaime Aparecido Cury (00000003-1046-5605); Celso Zilbovicius (0000-00020780-8120); Helder Henrique Costa Pinheiro (0000-0001-5567-3550); Helenita Corrêa Ely (0000-0002-5617-8436); Paulo Capel Narvai (0000-0003-4769-6896); Paulo Frazão (0000-00023224-0020).

\section{Agradecimentos}

Os autores agradecem ao Conselho Nacional de Desenvolvimento Científico e Tecnológico (CNPq) pelo fomento à pesquisa. Chamada Pública MCTI/ CNPq-MS/SCTIE/DECIT 10/2012 (Processo 403156/2012-2).

\section{Referências}

1. Savel TG, Foldy S. The role of public health informatics in enhancing public health surveillance. MMWR Suppl 2012; 61:20-4.

2. Asi YM, Williams C. The role of digital health in making progress toward Sustainable Development Goal (SDG) 3 in conflict-affected populations. Int J Med Inform 2018; 114:114-20.

3. França E, Teixeira R, Ishitani L, Duncan BB, Cortez-Escalante JJ, Morais Neto O, et al. Causas mal definidas de óbito no Brasil: método de redistribuição baseado na investigação do óbito. Rev Saúde Pública 2014; 48:671-81.

4. Cunha CC, Teixeira R, França E. Avaliação da investigação de óbitos por causas mal definidas no Brasil em 2010. Epidemiol Serv Saúde 2017; 26:19-30.

5. Garcia LP, Duarte E. 1a Conferência Nacional de Vigilância em Saúde: marco para a construção da Política Nacional de Vigilância em Saúde. Epidemiol Serv Saúde 2018; 27:e20180002.

6. World Health Organization. Health promotion glossary. Geneva: World Health Organization; 1998.

7. World Health Organization. Guidelines for drinking-water quality. Fourth edition. Geneva: World Health Organization; 2011.

8. Hunter JM, Rey L, Chu KY, Adekolu-John EO, Mott KE. Parasitic diseases in water resources development: the need for intersectoral negotiation. Geneva: World Health Organization; 1993.

9. Kumar JV. Is water fluoridation still necessary? Adv Dent Res 2008; 20:8-12.

10. Frazão P, Peres M, Cury J. Qualidade da água para consumo humano e concentração de fluoreto. Rev Saúde Pública 2011; 45:964-73.

11. Instituto Brasileiro de Geografia e Estatística. Pesquisa Nacional de Saneamento Básico, 2008. http://ibge.gov.br/home/estatistica/ populacao/condicaodevida/pnsb2008/default. shtm (acessado em 14/Fev/2017).

12. Coordenação Geral de Vigilância em Saúde Ambiental, Secretaria de Vigilância em Saúde, Ministério da Saúde. Programa Nacional de Vigilância em Saúde Ambiental Relacionada à Qualidade da Água para Consumo Humano. Brasília: Ministério da Saúde; 2005. (Série C. Projetos, Programas e Relatórios).

13. Frazão P, Narvai PC, organizadores. Cobertura e vigilância da fluoretação da água no Brasil: municípios com mais de 50 mil habitantes. São Paulo: Faculdade de Saúde Pública, Universidade de São Paulo; 2017.

14. Frazão P, Soares CCS, Fernandes GF, Marques RAA, Narvai PC. Fluoretação da água e insuficiências no sistema de informação da política de vigilância à saúde. Rev Assoc Paul Cir Dent 2013; 67:94-100.

15. Faculdade de Saúde Pública, Universidade de São Paulo. Consenso técnico sobre classificação de águas de abastecimento público segundo o teor de flúor. https://bit.ly/2L5SBie (acessado em 12/Dez/2015). 
16. Ministério da Saúde. Vigilância em saúde. http://portalms.saude.gov.br/vigilancia-emsaude/vigilancia-ambiental/vigiagua/sisagua (acessado em 03/Jul/2018).

17. Narvai PC. Cárie dentária e flúor: uma relação do século XX. Ciênc Saúde Colet 2000; 5:38192.

18. Frazão P, Ely HC, Noro LRA, Pinheiro HHC, Cury JA. O modelo de vigilância da água e a divulgação de indicadores de concentração de fluoreto. Saúde Debate 2018; 42:274-86.

19. Arretche M. Trajetória das desigualdades: como o Brasil mudou nos últimos cinquenta anos. São Paulo: Fundação Editora da Unesp; 2015.

20. Brasil. Lei no 6.050, de 24 de maio de 1974. Dispõe sobre a fluoretação da água em sistemas de abastecimento quando existir estação de tratamento. Diário Oficial da União 1974; 27 mai.

21. Barker LK, Duchon KK, Lesaja S, Robison VA, Presson SM. Adjusted fluoride concentrations and control ranges in 34 states - 2006-2010 and 2015. J Am Water Works Assoc 2017; 109:13-5.

22. Ministério da Saúde. Portaria no 1.469 , de 29 dezembro de 2000. Estabelece os procedimentos e responsabilidades relativos ao controle e vigilância da qualidade da água para consumo humano e seu padrão de potabilidade, e dá outras providências. Diário Oficial União 2001; 2 jan.

23. Conselho Nacional de Saúde. Resolução 588 de 12 de julho de 2018. Institui a Política Nacional de Vigilância em Saúde (PNVS). http:// conselho.saude.gov.br/web_cnvs/Reso588.pdf (acessado em 25/Set/2018).
24. Conselho Nacional de Saúde. 1a Conferência Nacional de Vigilância em Saúde: relatório final. https://goo.gl/cpgXc1 (acessado em 08/ Out/2018).

25. United States Environmental Protection Agency. National primary drinking water regulations. https://www.epa.gov/groundwater-and-drinking-water/national-primarydrinking-water-regulations (acessado em 08/ Out/2018).

26. Centers for Disease Control and Prevention. Water fluoridation reporting system. https:// www.cdc.gov/fluoridation/data-tools/report ing-system.html (acessado em 25/Set/2018).

27. Pelletier AR. Maintenance of optimal fluoride levels in public water systems. J Public Health Dent 2004; 64:237-9.

28. Association of State and Territorial Dental Directors. A report of state identified barriers to participation in the water fluoridation reporting system, 2003. https://www.astdd.org/ docs/final-wfrsreportwithcover-102720032. pdf (acessado em 16/Jun/2014).

29. Thacker SB, Qualters JR, Lee LM; Centers for Disease Control and Prevention. Public health surveillance in the United States: Evolution and Challenges. MMWR Suppl 2012; 61:3-9.

30. Zilbovicius C, Ferreira RGLA, Narvai PC. Água e saúde: fluoretação e revogação da Lei Federal no 6.050/1974. Rev Direito Sanit 2017; 18:104-24. 


\section{Abstract}

Sanitation data for 2008 indicated that $74.4 \%$ of Brazilian municipalities (counties) with more than 50,000 inhabitants were receiving fluoridated water, but no criteria were adopted to validate the information. The study aimed to verify the accuracy of information on water fluoridation, using as the reference water surveillance data from $\mathrm{mu}$ nicipalities with more than 50,000 inhabitants in 2008 and during 2010-2015. Data from different sources were used, comparing the information pertaining to the period. Water samples were collect$e d$, and fluoride concentration was obtained by the electrometric method. Verification was performed by comparing the data obtained from different sources. Data were compared for 601 (97.9\%) municipalities. The proportion of municipalities that performed surveillance based on external control increased from $39.4 \%$ to $48.5 \%$. There was a high rate of false positives and false negatives (15.1\%) in the data for 2008. Municipal coverage was $70.2 \%$, or 4.2 percentage points below the published estimate (74.4\%) for 2008. Surveillance records were observed in $54.3 \%$ of the fluoridated municipalities whose population coverage reached at least $50 \%$ of the population receiving treated water. There was an important improvement in fluoridation surveillance data, despite a high percentage of false positives and negatives. There are still wide interregional differences in the surveillance of water fluoridation in this sample of Brazilian municipalities, raising important public health challenges.

Fluoridation; Oral Health; Health Surveillance

\section{Resumen}

Los datos de saneamiento en 2008 indicaban que un 74,4\% de los municipios con más de 50 mil habitantes recibían agua fluorada, no obstante, no se adoptaron criterios para la validación de esta información. El estudio tuvo como objetivo verificar la exactitud de la información sobre la fluorización del agua, tomando como referencia datos de vigilancia del agua en municipios con más de 50 mil habitantes en el año 2008, y durante el período de 2010-2015. Se emplearon datos de diferentes fuentes comparando la información relativa a este período. Se recogieron muestras de agua y la concentración de fluoruro se obtuvo mediante un método electrométrico. El proceso de verificación se produjo por el contraste de datos obtenidos de diferentes fuentes. Se compararon los datos de 601 (97,9\%) municipios. La tasa de municipios que realizan vigilancia en base al heterocontrol pasó de un $39,4 \%$ a un $48,5 \%$. Se observó una elevada tasa de falsos positivos o negativos $(15,1 \%)$, en relación con los datos de 2008. La cobertura municipal fue de un 70,2\%, 4,2 puntos por debajo de la estimativa $(74,4 \%)$ divulgada en 2008. Se observaron registros de la práctica de vigilancia en un 54,3\% de los municipios fluorados, cuya cobertura poblacional alcanza a un 50\% o más de la población que recibe agua tratada. Hubo una sensible mejoría en los datos de vigilancia respecto al fluoruro, a pesar de que habia un porcentaje importante de falsos positivos y negativos. Todavía son grandes las diferencias interregionales relacionadas tanto respecto a la fluorización del agua, como a la implementación de vigilancia en los municipios investigados, implicando importantes desafíos para la salud pública.

Fluoruración; Salud Bucal; Vigilancia Sanitaria
Recebido em 27/Dez/2018

Versão final reapresentada em 18/Fev/2019 Aprovado em 28/Fev/2019 